\title{
DE QUELQUES FAIBLESSES DE LA DEFINITION TRADITIONNELLE DU CONTRAT
}

Depuis bientôt près d'un siècle ${ }^{1}$ les juristes de droit privé ont pris conscience que l'un de leurs instruments préférés, le contrat, était en crise, assailli qu'il était par une législation interventionniste croissante. Depuis lors, cette idée d'un contrat en crise n'a pas disparu de l'horizon doctrinal privatiste. En atteste la parution, à l'aube du $\mathrm{XXI}^{\mathrm{e}}$ siècle, d'un ouvrage intitulé «La nouvelle crise du contrat $»^{2}$ et qui, au moyen d'exemples et d'idées doctrinales certes renouvelés, s'intéresse lui aussi à la question du respect à accorder à la «loi des parties $"$.

L'ampleur de cette crise ne semble pas toutefois avoir été à ce jour nettement perçue par les auteurs qui l'ont identifiée, notamment parce que ceux-ci n'ont posé leur diagnostic qu'à partir d'une partie réduite du droit positif : le droit des contrats. Or, sitôt que l'on s'aventure dans d'autres matières, l'on prend conscience que la crise est bien plus grave et surtout qu'elle ne résulte pas tant du législateur, accusé d'empiéter sur le contrat en méconnaissant sa force obligatoire, que de l'instrument contractuel lui-même, dont plusieurs juristes doutent de la pertinence lorsqu'il est appliqué à plusieurs objets relevant de leur discipline. Ce n'est donc pas ici la force obligatoire du contrat qui est en crise, comme c'est le cas lorsque généralement les auteurs parlent de « crise du contrat » ${ }^{3}$, c'est le contrat lui-même.

Cette autre crise du contrat est semble-t-il apparue en droit public, à la fin du XIX ${ }^{\mathrm{e}}$ siècle — donc avant même le phénomène traditionnellement appréhendé au moyen de l'expression crise du contrat ${ }^{4}$-, à la suite des assauts dirigés contre le contrat par ces grands publicistes qu'ont été Maurice Hauriou, Léon Duguit, Gaston Jèze et Louis Rolland. En effet, lorsque ceux-ci ont voulu appréhender les relations d'un usager avec la personne en charge d'un service public, ils ont contesté que le contrat puisse être l'instrument adéquat pour cela ${ }^{5}$ et ont fini par emporter l'adhésion d'un Conseil d'État jusque-là plutôt favorable au contrat ${ }^{6}$. Par la suite, la contestation du contrat a progressivement gagné d'autres matières où les auteurs ont pu récuser dans telle ou telle situation la pertinence de l'analyse contractuelle. C'est ainsi par exemple que les travaillistes ont un temps été tentés par une contestation de l'analyse

\footnotetext{
${ }^{1}$ C'est à partir des années 1930 que la doctrine a commencé à parler de «crise du contrat » pour désigner l'empiètement du législateur sur la « loi des parties ». L'une des premières occurrences de l'expression paraît se situer dans la préface de Gabriel Marty à la thèse d'É. de Lagrange, La crise du contrat et le rôle du juge, Librairie du Recueil Sirey, 1935. La thèse, en tant que telle, ne traite pas de la crise du contrat telle que nous l'entendons actuellement mais de la question du changement de circonstances. En revanche, dans sa préface, G. Marty indique que la crise du contrat pourrait prendre à l'avenir un autre aspect si l'article 1134 continuait à voir son importance décroître (p. III) et cette fois c'est bien l'acception actuelle de l'expression qui est retenue.

${ }^{2}$ C. Jamin et D. Mazeaud (dir.), La nouvelle crise du Contrat, Dalloz, 2003.

${ }^{3}$ A. Barreyre, L'évolution et la crise du contrat : Étude synthétique et critique, M. Laborde-Lacoste (dir.), thèse de doct., Université de Bordeaux, 1937, Bière, p. 1, où l'on peut lire ces mots : « L'article 1134 du Code civil se meurt, l'article 1134 est mort ». Rappr. Ch. Jamin, «Quelle nouvelle crise du contrat ? : Quelques mots en guise d'introduction », in La nouvelle crise du contrat, C. Jamin et D. Mazeaud (dir.), Dalloz, 2003, p. 21 ; J.-P. Chazal, « De la théorie générale à la théorie critique du contrat », RDC, 2003, 1.

${ }^{4}$ Cf. supra note 1.

${ }^{5}$ Not. L. Duguit, « De la situation des particuliers à l'égard des services publics », RDP, 1907, p. 411-439, spé. p. $428 \mathrm{~s}$.

${ }^{6}$ Retenant l'analyse contractuelle : CE, Rosier et Winkel, 7 août 1909, DP, 1911, 3, p. 7. Adde, pour la doctrine officielle, É. Lafferière, Traité de la juridiction administrative et des recours contentieux, $2^{\mathrm{e}}$ éd., Berger-Levrault, 1896, p. 616 ou encore les conclusions de Jean Romieu sous CE, Syndicat Croix de Seguey-Tivoli, 21 déc. 1906, Rec. Lebon et $S ., 1907,3$, p. 37.
} 
contractuelle tant pour les relations individuelles de travail que collectives ${ }^{7}$, puis que les spécialistes du droit de la santé, sous l'influence du droit public, ont également commencé à rejeter l'analyse contractuelle de la relation médicale ${ }^{8}$. Même des matières plus " mercantiles », a priori donc moins hostiles au contrat, ont également contesté la qualification contractuelle de certains de leurs objets. Tel a été le cas pour le droit de la concurrence $^{9}$, à propos de la qualification des ententes anti-concurrentielles ou encore, dans la même veine, du droit des marchés financiers, à propos de la qualification des actions de concert $^{10}$. Cette contestation du contrat, parce qu'elle est notamment liée à la difficulté d'admettre la qualification contractuelle en présence d'actes sciemment illicites où, par définition, les parties n'ont pas l'intention de créer des effets, n'est pas sans rappeler celle qu'on retrouve en droit pénal. En effet, les pénalistes contestent généralement la pertinence des définitions civilistes du contrat lorsqu'ils ont à rechercher, afin d'établir la matérialité d'une infraction, si tel fait est ou non constitutif d'un contrat en droit pénal ${ }^{11}$. La contestation est encore plus vigoureuse lorsqu'il s'agit d'analyser les rapports pouvant se nouer entre une juridiction d'une part et un prévenu ou un condamné d'autre, lorsque ce dernier consent à reconnaître préalablement sa culpabilité ou consent aux peines ou aménagement de peine, tel le travail d'intérêt général, pour lesquels son accord est nécessaire ${ }^{12}$.

Ce rejet du contrat doit interroger le civiliste et plus généralement le théoricien du droit épris de concepts généraux susceptibles de s'appliquer dans l'ensemble du droit. Comment se fait-il que le concept de contrat soit contesté dans ces matières ? L'explication réside selon nous dans l'existence de faiblesses théoriques du contrat, faiblesses qui à ce jour n'ont pas été bien perçues par les juristes ou, lorsqu'elles l'ont été, ont été sciemment ignorées afin de ne pas perturber un édifice intellectuel construit par des générations de juristes et que l'on craindrait d'ébranler.

Cette attitude ne nous semble plus aujourd'hui acceptable. Si elle apporte assurément aux civilistes un certain confort, puisqu'en fermant les yeux sur les difficultés théoriques du

${ }^{7}$ H. Batiffol, « La crise du contrat et sa portée », $A P D, 1968$, vol. XIII, p. 14 et, plus largement, G. Lyon-Caen, « Défense et illustration du contrat de travail », APD, 1968, vol. XIII, p. 59-69.

${ }^{8}$ D. Thouvenin, « La disponibilité du corps humain : corps sujet ou corps objet ? », Actes, 1985, 49/50 ou, plus récemment, M. Girer, Contribution à une analyse rénovée de la relation de soins : Essai de remise en cause du contrat médical, F. Vialla (dir.), thèse de doct., 2008, LEH.

9 Bulletin de la Société d'Études législatives, 1932, Question n88, Commission des Ententes économiques, Rapport présenté au nom de la Commission par M. Georges Ripert, p. 253-266, spé. p. 257 où G. Ripert, manifestement influencé par les concepts issus du droit public, affirme que l'entente « est règlement plus que contrat, statut plus que convention ». Cf., plus récemment, Y. Guenzoui, La notion d'accord en droit privé, C. Hannoun (dir.), thèse de doct., Université de Cergy-Pontoise, 2009, LGDJ, spé. n ${ }^{\text {os }} 54$ et 107 où cette fois l'analyse contractuelle, issue du droit privé, est concurrencée par une analyse économique, issue du droit américain, en particulier du Sherman Act de 1890.

${ }^{10}$ M.-A. Frison-Roche, «Esquisse d'une sociologie du droit boursier », L'Année sociologique, vol. 49, 1999 , $\mathrm{n}^{\circ} 2$, p. 457 s. ; F. Castres Saint-Martin - Drummond, « Le contrat comme instrument financier » in L'avenir du droit, Mélanges en hommage à François Terré, Dalloz, PUF, Éditions du JurisClasseur, 1999, p. 668 ; A.-C. Muller, Droit des marchés financiers et droit des contrats, H. Synvet (dir.), thèse de doct., Université PanthéonAssas, 2007, Économica, $\mathrm{n}^{\circ} 545$; Y. Guenzoui, op. cit., $\mathrm{n}^{\mathrm{o}} 64$; O. Dexant - de Bailliencourt, Les pactes d'actionnaires dans les sociétés cotées, H. Synvet (dir.), thèse de doct., 2012, Dalloz, $\mathrm{n}^{\mathrm{os}} 306 \mathrm{~s}$.

${ }^{11} \mathrm{M}$. Vasseur, « Des effets en droit pénal des actes nuls ou illégaux d'après d'autres disciplines », RSC, 1951 ou $\mathrm{X}$. Pin, Le consentement en matière pénale, P. Maistre du Chambon (dir.), thèse de doct., Université de Grenoble, 2002, LGDJ, ${ }^{\text {os }} 691$ : « La notion de contrat, bien que celui-ci ait été qualifiée de "pillier" du droit, est fondamentalement inconciliable avec le droit pénal ». Comp. E. Palvadeau, Le contrat en droit pénal, V. Malabat (dir.), thèse de doct., Bordeaux IV, 2011.

${ }^{12}$ M. Danti-Juan, « Le consentement et la sanction » in Mélanges offerts à P. Couvrat : la sanction du droit, PUF, 2001, p. 377 ; M. van de Kerchove, "Contractualisation de la justice pénale ou justice pénale contractuelle? » in La contractualisation de la production normative, S. Chassagnard-Pinet et D. Hiez (dir.), Dalloz, 2008, p. 192. 
contrat, ceux-ci peuvent continuer à l'utiliser comme si de rien n'était, cette attitude n'en est pas moins contestable au regard des difficultés pratiques qu'elle entraîne pour les acteurs du droit et qui imposent à nos yeux une indispensable refonte de la définition du contrat.

\section{LES DIFFICULTES THEORIQUES}

Communément, le contrat est défini comme un accord de volontés entre deux ou plusieurs personnes destiné à créer des obligations. En outre, il est souvent précisé, en amont de cette définition, que le contrat est une espèce d'un genre plus général, l'acte juridique, espèce qui en côtoie d'autres et notamment l'acte unilatéral. Cette définition traditionnelle du contrat se trouve reprise dans les textes nouveaux du code civil. Ainsi, après que l'article 1100-1 du code civil a indiqué que l'acte juridique peut être "conventionnel ou unilatéral », l'article 1101 du même code reprend la définition précitée du contrat, en apportant toutefois cette précision que le contrat peut aussi transmettre ou éteindre des obligations, façon d'indiquer que la distinction du contrat et de la convention, construite à partir du droit romain tardif, n'a plus désormais cours ${ }^{13}$. Cette approche du contrat est pourtant problématique tant dans sa définition positive que dans sa définition négative, c'est dire dans la manière dont le contrat s'articule avec d'autres concepts voisins, en particulier l'offre, l'acceptation, l'acte juridique et l'acte unilatéral. En effet, il apparaît à l'examen que la définition positive est inutilisée et que la définition négative est erronée ou à tout le moins contestable.

\section{Une définition positive inutilisée}

Soutenir que la définition traditionnelle du contrat n'est pas utilisée peut au premier abord surprendre. Pourtant, un examen minutieux des cas dans lesquels les acteurs du droit ou de la science du droit se posent la question de la qualification contractuelle d'un objet révèle qu'ils n'y répondent pas toujours, loin s'en faut, en usant de cette définition.

Premièrement, il peut arriver que les juges écartent l'un des critères de la définition traditionnelle du contrat, à savoir l'intention de créer des effets de droit. Parce que phénomène se manifeste dans des contextes fort différents (droit des contrats, droit du travail, droit commercial, droit de l'arbitrage, droit international public) et de manière durable dans le temps ${ }^{14}$, il ne saurait être ignoré. On en donnera ici un exemple bien connu : la convention d'assistance bénévole. L'examen des arrêts dans lesquels la Cour a accepté de qualifier de contrat les actes par lesquels une personne a bénévolement porté assistance à autrui révèle que cette qualification est souvent retenue alors même qu'il n'existe aucune intention de créer des effets de droit. Généralement, les auteurs ont tendance à critiquer ces décisions ${ }^{15}$ en considérant qu'elles reposeraient sur un artifice : la Cour de cassation affirmant selon eux l'existence d'une intention de créer des effets de droit qui serait manifestement inexistante. C'est là toutefois ne pas comprendre ce qui semble être le discours dominant de la Cour de cassation dans ces arrêts laquelle, loin de créer une fiction, paraît davantage refuser la définition traditionnelle du contrat, centrée sur l'intention de créer des effets de droit, et dont se prévalent les plaideurs.

Ce rejet de la définition traditionnelle du contrat est particulièrement manifeste dans une décision de 1962, intervenue sur un arrêt d'appel ayant refusé la qualification contractuelle de

\footnotetext{
${ }^{13}$ L'on enseignait traditionnellement que le contrat ne faisait que créer des obligations là où la convention pouvait aussi en étendre ou en transmettre.

${ }^{14}$ En ce sens Contrat ou acte juridique? Étude à partir de la relation médicale, D. Fenouillet (dir.), 2016 Partie 1, Titre 1, Chapitre 1, Section 1, §1, A.

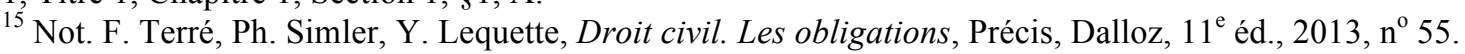


l'assistance bénévole au vu de l'absence d'une intention de créer des effets de droit ${ }^{16}$. Dans cet arrêt de 1962, la Cour de cassation censure la décision d'appel, non pas en considérant que les faits auraient été dénaturés - la cour d'appel aurait refusé l'existence d'une intention de créer des effet de droit alors qu'une telle intention existerait manifestement -, mais en considérant que la loi a été violée ${ }^{17}$. Ce faisant, la Cour de cassation affirme que la définition retenue par la cour d'appel n'est pas celle prévue par la « loi », autrement dit que la cour d'appel a retenu une définition du contrat qui n'est pas exacte et qui l'a conduite à rejeter à tort l'existence d'un tel contrat.

Deuxièmement, il peut arriver que des critères soient non pas retranchés de cette définition traditionnelle mais au contraire ajoutés. Le phénomène se rencontre fréquemment chez les auteurs spécialistes dans des matières autres que le droit des contrats (droit public, droit de la famille, droit du travail, droit de la concurrence, droit pénal, droit médical, droit des marchés financiers) ${ }^{18}$ et il peut aussi parfois s'observer chez les magistrats ${ }^{19}$. On l'illustrera ici à partir du droit de la santé où il est particulièrement manifeste. En effet, nombre d'auteurs spécialistes de cette matière contestent la qualification contractuelle de la relation médicale en avançant des arguments tirés du caractère peu économique de cette relation, du peu de liberté dont disposent les parties pour aménager leur relation, de la possibilité pour le patient de révoquer son consentement ou encore de l'absence de formalisme $^{20}$. Or, aucun de ces critères ne figure dans la définition traditionnelle du contrat. Certes, le contrat sert souvent d'instrument d'échange économique, mais il assure également une fonction d'organisation sociale ${ }^{21}$. Certes, également, le contrat nécessite une part de liberté, puisqu'il faut bien consentir, mais il suffit d'avoir la liberté de conclure un contrat, quand bien même son contenu serait entièrement déterminé par un tiers, pour que le contrat existe $^{22}$. En atteste par exemple la récente reconnaissance du caractère contractuel du contrat d'adhésion ${ }^{23}$. Certes, encore, nombre de contrats importants sont conclus par écrit, mais il n'en demeure pas moins que le consensualisme impose une liberté des formes, ce que reconnaît l'article 1102 nouveau du code civil ${ }^{24}$. Certes, enfin, le contrat est un acte obligatoire puisqu'il crée des obligations ; cependant obligatoire ne veut pas dire irrévocable. De plus en plus de contrats (consommation, immobilier, etc.) comprennent des possibilités de

\footnotetext{
${ }^{16}$ Cass., $2^{\mathrm{e}}$ civ., 23 mai 1962, Bull., II, no 459. Comp. ant. Cass., sect. civ., 27 mai 1959, Bull., I, n 271, p. 225 qui paraît en revanche s'ancrer dans la définition traditionnelle du contrat et qui, de facto, dénature quelque peu les faits de l'espèce. Des arrêts postérieurs à 1962 sont également un peu ambigus, sans être néanmoins inconciliable avec l'analyse ici proposée, not. Cass., com., 25 sept. 1984, n 83-12.666, Bull., IV, n ${ }^{\circ} 242$ ou Cass., $1^{\text {re }}$ civ., 3 janv. 1991, $\mathrm{n}^{\circ} 88-19.901$.

${ }^{17}$ Alors que la cour d'appel avait rejeté la qualification de contrat — pour elle il « s'agissait d'un coup de main, acte d'entraide ou service gratuit », semblant suggérer par là que les parties n'avaient pas eu l'intention de créer des effets de droit mais avaient simplement voulu agir par devoir de courtoisie —, la Cour de cassation a affirmé « qu'en refusant ainsi de reconnaître à l'accord de volontés [...] les caractères d'une convention d'assistance [...], les juges du second degré ont violé les dispositions précitées [art. 1134 c. civ. et art. 7 de la loi du 20 avr. $1810] »$.

${ }^{18} \mathrm{Cf}$. Contrat ou acte juridique, op. cit., Introduction, Section $1, \S 1$.

${ }^{19} \mathrm{Cf}$. infra à propos de l'analyse portée par le juge administratif sur la qualification de la nature des relations entre l'usager d'un service public administratif et la personne en charge de ce service.

${ }^{20}$ Cf. Contrat ou acte juridique, op. cit., Chapitre préliminaire, Section 1.

${ }^{21}$ P. Didier, « Brèves notes sur le contrat-organisation » in Mélanges en l'honneur de François Terré : L'avenir $d u$ droit, Dalloz, PUF et Éditions du JurisClasseur, 1999 ; M. Mekki, « Le discours du contrat. Quand dire, ce n'est pas toujours faire ", RDC, 2006, p. 299. J. Rochfeld, Les grandes notions du droit privé, PUF, 2012, p. 450-453.

${ }^{22}$ Rappr. H. Batiffol, « La crise du contrat et sa portée », précité, p. 22.

${ }^{23}$ Art. 1110 al. 2 nouv. c. civ. : «Le contrat d'adhésion est celui dont les conditions générales, soustraites à la négociation, sont déterminées à l'avance par l'une des parties. »

${ }^{24}$ « Chacun est libre de [...] déterminer le contenu et la forme du contrat dans les limites fixées par la loi. »
} 
rétractation et pourtant ils n'en demeurent pas moins des contrats, ce que confirme au demeurant le nouvel article 1122 du code civil prévoyant en droit commun du contrat un délai de rétractation ${ }^{25}$.

Si ces critères extérieurs à la définition du contrat sont utilisés par les auteurs c'est parce que, trop souvent, lorsque se pose la question de savoir si tel objet est ou non un contrat, les auteurs répondent à cette question non pas en mobilisant les seuls éléments contenus dans la définition du contrat, mais en faisant appel aux éléments qui leur sont donnés par l'image commune du contrat, autrement dit - pour reprendre un vocabulaire emprunté à la psychologie sociale - par la représentation sociale du contrat. En effet, la structure de la représentation sociale du contrat laisse apparaître que les quatre éléments précités - échange économique, liberté, formalisme et irrévocabilité - y occupent une place très importante ${ }^{26}$. Ceci tient au fait que cette représentation sociale du contrat est construite à partir de l'expérience que les juristes ont du contrat dans leur quotidien, expérience qui paraît nourrie de deux types sociologiques: d'un côté les "petits contrats» et de l'autre les " grands contrats », pour reprendre une typologie esquissée par Jean Carbonnier et François Terré27. Les «petits contrat», c'est-à-dire ceux que les juristes concluent plusieurs fois par jours (vente alimentaire, prestation de services divers), conduisent les juristes à intégrer à leur représentation sociale du contrat les idées d'échange économique et de liberté (puisqu'une grande liberté règne dans la conclusion de ces contrats du quotidien). Quant aux " grands contrats ", c'est-à-dire les contrats conclus aux moment importants de la vie (travail, logement, prêt, mariage), ceux-ci conduisent les juristes à intégrer à la représentation sociale du contrat l'idée que ce dernier serait un instrument formaliste et irrévocable. Par ailleurs, le fait que les juristes recourent fréquemment à cette représentation sociale du contrat plutôt qu'à sa seule définition juridique est un phénomène naturel. Les travaux en psychologie sociale montrent en effet que lorsque des personnes sont confrontées à un problème particulier, elles résolvent ce problème avant tout à partir de leur représentation sociale de l'objet en cause. Dès lors, le fait que le contrat soit à la fois un objet d'étude dans leur activité scientifique et un objet dont ils se servent pour leur vie quotidienne, perturbe l'analyse juridique et aboutit à cette conséquence que la définition traditionnelle du contrat est trop souvent inutilisée. Telle n'est pas la seule faiblesse du contrat. Il apparaît également que celui-ci est théoriquement fragile lorsqu'on examine sa définition négative, c'est-à-dire la manière dont il s'articule avec des concepts voisins, appartenant à une même famille pour reprendre une métaphore utilisée jadis par Charles Jarrosson ${ }^{28}$.

\section{Une définition négative contestable}

La manière dont le contrat se trouve traditionnellement articulé avec les concepts dont il est proche suscite quelques interrogations quant à l'opportunité voir l'exactitude de ces analyses. Montrons-le pour ce qu'on pourrait appeler, en filant la métaphore familiale du contrat, les concepts filiaux d'offre et d'acceptation, le concept parental d'acte juridique et le concept collatéral d'acte unilatéral

\footnotetext{
${ }^{25}$ « La loi ou le contrat peuvent prévoir [...] un délai de rétractation, qui est le délai avant l'expiration duquel son bénéficiaire peut rétracter son consentement. »

${ }^{26}$ Sur le détail de cette représentation sociale du contrat cf. Contrat ou acte juridique, op. cit., Chapitre préliminaire, Section 2.

${ }^{27}$ J. Carbonnier, «Sociologie de la vente », Travaux et conférences de 1'Université Libre de Bruxelles, 1960, vol. VIII, p. 150-153 (reproduit dans Écrits de Jean Carbonnier, PUF, 2008, p. 521-525) ; F. Terré, "Sur la sociologie juridique du contrat », APD, 1968, p. 80 ; J. Carbonnier, Flexible droit, LGDJ, 2001, p. 339.

${ }^{28}$ Ch. Jarrosson, La notion d'arbitrage, B. Oppetit (préf.), LGDJ, 1987.
} 
Si l'on s'intéresse d'abord aux concepts filiaux du contrat que sont l'offre et l'acceptation, il apparaît à l'examen qu'il n'est pas exact de prétendre que le contrat résulterait de la rencontre d'une offre et d'une acceptation. Dire cela impliquerai en effet une chronologie donnée (d'abord l'offre puis l'acceptation), ainsi qu'une certaine répartition des rôles (l'offrant d'un côté, l'acceptant de l'autre). Or, comme l'ont déjà relevé plusieurs auteurs $^{29}$ dont les idées ont parfois percé dans des instruments de codification privés ${ }^{30}$, ni cette chronologie ni cette répartitions des rôles ne se rencontrent systématiquement en pratique. Il suffit pour s'en rendre compte d'examiner l'hypothèse suivante.

Soit deux ou plusieurs personnes morales - des entreprises, des États, peu importe désireuses de passer entre elles un contrat particulièrement complexe. En vue de la signature de ce contrat, elles désignent chacune des représentants chargés des négociations. Une fois un projet d'accord établi par ces négociateurs, ceux-ci adressent conjointement aux personnes morales le projet afin qu'elles expriment leur volonté sur celui-ci, étant entendu que le contrat n'entrera en vigueur qu'en cas d'accord de toutes les parties à la négociation. Au moment où ces personnes morales se prononcent, elles le font sans respecter entre elles un ordre ni même sans savoir si leur éventuel futur cocontractant s'est déjà prononcé. Dans cet exemple, qui n'est pas seulement imaginaire et qui se rencontre dans la pratique contractuelle, en particulier internationale $^{31}$, il apparaît clairement qu'aucune des parties au contrat n'a formulé d'offre ou d'acceptation. Toutes ont, par la voie de leurs représentants, élaboré l'offre et toutes l'ont également accepté (ou refusé). Où l'on voit bien qu'un tel contrat ne s'est pas formé par la rencontre d'une offre et d'une acceptation, mais par la rencontre de deux acceptations d'un projet conjointement élaboré. Dans ces conditions, il est pour le moins inexact d'affirmer ce que fait pourtant l'article 1113 nouveau du code civil — que « [1]e contrat est formé par la rencontre d'une offre et d'une acceptation par lesquelles les parties manifestent leur volonté de s'engager ». En réalité, si l'on voulait conserver la trame de cet article - mais la formule ne serait alors guère élégante - , il faudrait écrire que « [1] e contrat est formé par la rencontre des manifestations de volonté de deux parties de s'engager. »

Le caractère inapproprié de cette définition négative apparaît également lorsqu'on s'intéresse aux concepts collatéraux du contrat, en particulier à l'acte unilatéral. En effet, le contrat et l'acte unilatéral sont perçus comme désignant tant une certaine procédure créatrice de normes que les effets de cette procédure ${ }^{32}$. Ainsi, quand il est question de contrat, cela peut désigner tant l'accord des volontés, que les normes issues de cette accord. Or, cette

\footnotetext{
${ }^{29}$ A. L. Corbin, « Offer and Acceptance, and Some of the Resulting Legal Relations », The Yale Law Journal, 1917, 3, url : http://www.jstor.org/stable/786706, p. 183 ; M. Fontaine, « Offre et acceptation, approche dépassée du processus de formation des contrats ? » in Mélanges offert à Pierre Van Ommeslaghe, Bruylant, $2000, \mathrm{n}^{\mathrm{os}} 6$ et 7 ; F. Limbach, Le consentement contractuel à l'épreuve des conditions générales des contrats. De l'utilité du concept de la déclaration de volonté, C. Witz et S. Peruzetto (dir.), thèse de doct., Université Toulouse 1 et Université de la Sarre, 2004, LGDJ, n 111 ; M. Fontaine, «La théorie de la formation du contrat et la pratique $\mathrm{du}$ "closing" ", in Mélanges en l'honneur de Camille Jauffret-Spinosi, Dalloz, 2013, $\mathrm{n}^{\circ} 16$

${ }^{30}$ Art. 2.1.1. des Principes Unidroit indiquant : « Le contrat se conclut soit par l'acceptation d'une offre, soit par un comportement des parties qui indique suffisamment leur accord. » Adde l'art. II.-4:211 du DCFR, intitulé : "Contrats non conclus au moyen d'une offre et d'une acceptation ».

${ }^{31} \mathrm{Cf}$. les articles précités de M. Fontaine.

${ }^{32}$ Pour le contrat, cf. not. H. Kelsen, « La théorie juridique de la convention », Archives de philosophie du droit et de sociologie juridique, 1940, url : http://gallica.bnf.fr/ark:/12148/bpt6k888152j/f327.image, p. 36; G. Rouhette, Contribution à l'étude critique de la notion de contrat, R. Rodière (dir.), thèse de doct., Paris, 1965, not. $\mathrm{n}^{\text {os }} 91$ et 115 . Pour l'ensemble des sources, cf. C. Thibierge, « Sources du droit, sources de droit : une cartographie » in Libres propos sur les sources du droit. Mélanges en l'honneur de Philippe Jestaz, Dalloz, 2006, p. 519-548, spé. p. 529. Adde, pour le traité international, E. Bourdoncle, « Fitzmaurice et la distinction entre traité et obligation conventionnelle » in Grandes pages du droit international. Les sources, Pedone, 2016, p. 55-86, spé. p. 60-62.
} 
ambivalence est problématique car elle empêche d'appréhender correctement les actes juridiques qui combineraient à la fois des effets contractuels et des effets unilatéraux. Prenons par exemple une convention collective ou encore une concession de service public. Ces deux opérations produisent des effets contractuels entre les parties elles-mêmes (par exemple, dans le premier cas, la création d'une redevance versée à la personne publique et dans le deuxième cas une obligation de renégociation), mais elles produisent également des effets unilatéraux à l'égard de tiers non signataires de l'acte (les travailleurs et les employeurs dans le premier cas, les usagers du service public concédé dans le second). Appréhender ces actes au moyen de l'approche traditionnelle du contrat et de son articulation avec l'acte unilatéral est mission impossible compte tenu de l'ambivalence de ces concepts et de leur caractère opposé. L'ambivalence de ces concepts implique en effet que la qualification contractuelle ou unilatérale ne sera retenue que si la procédure et les effets correspondent à ces deux espèces d'acte juridique. Or, ceci n'est pas le cas dans les exemples donnés : certes, par leur procédure, la convention collective et la concession semblent se rapprocher du contrat mais, par leurs effets, ils sont proches tant du contrat que de l'acte unilatéral et ce dualisme ne saurait être nié ${ }^{33}$. Dès lors, ces deux exemples ne peuvent pas être seulement contractuels ou unilatéraux. Peuvent-ils pour autant être les deux à la fois ? L'idée est parfois avancée par la doctrine qui propose alors de retenir une qualification « mixte ${ }^{34}$ ou « hybride ${ }^{35}$. Mais là encore la solution n'est pas satisfaisante car elle repose sur l'idée que le contrat et l'acte juridique pourraient être "fusionnés" alors qu'en raison de leur caractère opposé, cette fusion n'est pas envisageable. Où l'on voit que l'ambivalence des concepts de contrat et d'acte unilatéral, ainsi que leur caractère opposé, empêchent d'appréhender correctement les exemples précités.

Le seul moyen pour appréhender correctement ces exemples serait de procéder à une réduction sémantique des concepts en cause pour que ceux-ci ne soient plus ambivalents. Si le contrat et l'acte unilatéral désignaient seulement certains effets de droit - leur composante procédurale étant alors appréhendée au moyen du concept d'acte juridique - alors il serait possible d'appréhender correctement la concession et la convention collective. Il suffirait alors de voir dans la convention collective et la concession le résultat de plusieurs actes juridiques correspondant aux actes volontaires des personnes parties à ces opérations. Il faudrait en outre ajouter que ces actes juridiques créent des effets différents : les uns sont contractuels, les autres sont unilatéraux (réglementaires). Les qualificatifs « contractuel» et « unilatéral » portant sur des objets différents (des normes distinctes), il n'y aurait plus de fusion à faire entre des qualifications opposées. Cette solution permettrait en outre de lutter contre une autre faiblesse théorique et relative à l'articulation de celui-ci avec le concept d'acte juridique.

Le caractère inapproprié de la définition négative du contrat apparaît enfin lorsque le contrat se trouve mis en rapport avec son concept parent, l'acte juridique, concept dont il est souvent dit qu'il serait un genre dont le contrat serait une espèce. Une telle affirmation apparaît problématique lorsqu'on confronte la définition traditionnelle de l'acte juridique avec celle du contrat. Traditionnellement l'acte juridique est défini comme une ou plusieurs manifestations de volonté créatrices d'obligations ou et cela nous semble presqu'équivalent, d'effets de droit, de normes. Dès lors, affirmer que le contrat serait un acte juridique impliquerait que celui-ci se caractériserait lui aussi par une ou plusieurs volontés créatrices de

\footnotetext{
${ }^{33}$ Comp. J. Dufau, « La nature juridique de la concession de service public », in Mélanges René Chapus : Droit administratif, Montchrestien, 1992, qui tente de soutenir la seule qualification contractuelle de la concession.

${ }^{34} \mathrm{Y}$. Madiot, Aux frontières du contrat et de l'acte administratif unilatéral : recherches sur la notion d'acte mixte en droit public français, J. Lamarque (dir.), thèse de doct., Université de Poitiers, 1971, LGDJ.

${ }^{35}$ J. Pélissier, A. Supiot et A. Jeammaud, Droit du travail, $24^{\mathrm{e}}$ éd., Dalloz, 2008, nº 1037.
} 
normes. A priori, cela ne pose guère de difficultés puisque, nous venons de l'indiquer, le contrat résulte de deux manifestations de volonté - l'offre et l'acceptation - dont l'ont dit parfois, un peu confusément, qu'elles fusionneraient pour créer des effets de droit ${ }^{36}$. Mais, et c'est là que le doute vient marquer son emprise sur la pensée, est-on bien sûr que ces deux manifestations de volonté formant le contrat créent un acte juridique lorsqu'elles se « rencontrent » ? Est-on bien sûr qu'avant cette transmutation alchimique il n'y a rien ?

Traditionnellement, tant dans la doctrine française ${ }^{37}$ qu'allemande $^{38}$, il est répondu en tout ou partie négativement à cette question, au motif que l'offre ne créerait en principe par elle-même aucun effet de droit et qu'elle ne pourrait pas en outre être révoquée. Le point mérite cependant d'être discuté en usant pour cela d'une conception plus précise de l'effet de droit, conception moins axée sur l'existence d'un effet obligatoire, immédiatement tangible, que sur la seule existence d'une norme ${ }^{39}$, indépendamment de son entrée en vigueur ou de son caractère révocable ${ }^{40}$. En effet, si l'on reprend notre exemple précédent du contrat formé avec l'aide de négociateurs, lorsque chacune des parties signe le projet de contrat qui lui est adressé, elle ne s'engage évidemment qu'à la condition que l'autre s'engage également. Techniquement, l'engagement de chacune des parties suit la structure suivante : d'une part, cet engagement crée une norme (un modèle de conduite) dont le signataire est l'assujetti et le cocontractant le bénéficiaire ; d'autre part, cette norme se trouve conditionnée pour sa vigueur à l'existence d'une autre norme que le cocontractant doit lui aussi prendre pour lui même. Autrement dit: l'un s'engage à condition que l'autre s'engage. Telle est la structure caractéristique des normes contractuelles. Or, l'opération contractuelle ainsi comprise, il apparaît nettement qu'elle repose sur deux actes juridiques, puisque chaque signature manifeste la volonté de son auteur d'une part et crée une norme, certes non encore entrée en vigueur mais qui n'en est pas moins existante, d'autre part. La seule chose que déclenche la « rencontre des volontés », c'est l'entrée en vigueur de la norme. Mais, finalement, point d'alchimie ici, point de rencontre mystérieuse des volontés : il y a seulement survenance d'un fait juridique déclenchant la mise vigueur d'une norme préexistante ; c'est cela que renferme cette «boîte noire » qu'est la rencontre des volontés ${ }^{41}$. Ainsi, dans une telle approche, les manifestations de volonté dont le contrat est formé sont elles-mêmes des actes juridiques et elles comprennent en outre déjà tous les effets du contrat. Dès, il ne semble plus possible d'affirmer que le contrat pourrait être en soi son propre acte juridique créant ses propres effets de droit. Il apparaît plus simple de dire - trois actes juridiques (offre, acceptation, contrat) c'est trop ! - que le contrat est seulement le résultat d'une succession d'actes juridiques. Il n'est pas un acte juridique. La conclusion peut heurter la tradition juridique, mais on ne saurait la refuser pour ce seul motif, alors qu'elle constitue semble-t-il l'application la plus rigoureuse et la plus simple des principes en présence. Refuser cette analyse c'est à coup sûr

\footnotetext{
${ }^{36}$ Rappr. Cass. com., 13 oct. 1998, n 96-13.107 où l'on peut lire dans le moyen « qu'un contrat n'est formé que par la rencontre de volontés qui fusionnent dans un consentement mutuel qui suppose une offre et une acceptation de cette offre $\gg$.

37 J.-L. Aubert, Notions et rôles de l'offre et de l'acceptation dans la formation du contrat, J. Flour (dir.), Université de Paris, 1970, LGDJ, spé. n ${ }^{\text {os }} 195$ et 329.

${ }^{38}$ F. Limbach, op. cit., $\mathrm{n}^{\circ} 181$.

${ }^{39}$ Sur l'intérêt du mot norme, cf. Contrat ou acte juridique, op. cit. Partie 1, Titre 2 Chapitre 2, Section 1, $\$ 4$.

${ }^{40}$ Il faut être conscient en effet que si l'on en vient à exiger que la norme soit en vigueur pour qu'un effet de droit existe, alors il faudrait refuser la qualité d'acte juridique aux contrats assortis d'une condition suspensive ou encore aux actes administratifs ou législatifs dépendant pour leur entrée en vigueur de l'édiction d'un autre acte administratif. De même, subordonner la qualification d'acte juridique à l'exigence que la norme ne puisse pas être révoquée imposerait aussi de refuser cette qualité à tous les contrats assortis d'une faculté de révocation, ainsi qu'à l'ensemble des actes administratifs, puisque ceux-ci peuvent être retirés dans un délai de quatre mois après leur édiction (art. L. 243-4 code des relations entre le public et l'administration).

${ }^{41}$ Expression employée par R. Libchaber, « Réflexions sur les effets du contrat » in Mélanges offerts à Jean-Luc Aubert, Dalloz, 2005, $\mathrm{n}^{\circ}$ 6. L'auteur parle également de « mystère ».
} 
laisser perdurer certaines des difficultés pratiques auxquelles sont exposés les utilisateurs du contrat car, comme l'affirmait naguère Voirin, « les principes se vengent souvent du dédain qu'on leur témoigne ${ }^{42}$.

\section{DES DIFFICULTES PRATIQUES}

L'ensemble des difficultés théoriques que nous avons dégagées sont source d'autres difficultés pratiques qui, certes, ne sont pas systématiques, mais existent néanmoins.

Premièrement, les faiblesses théoriques de la définition traditionnelle du contrat identifiées plus haut conduisent à une complexification importante, voir à une inintelligibilité du droit français. S'agissant de la complexification, l'exigence d'une intention de créer des effets de droit a par exemple conduit les spécialistes du droit de la concurrence, des marchés financiers ou du droit médical à refuser l'application du concept contractuel dans leur matière $^{43}$. À leurs yeux, les parties à une entente, une action de concert ou encore une relation médicale ne seraient pas mues par une intention de créer des effets de droit, de sorte que ces relations ne sauraient être appréhendées au moyen du concept de contrat. D'autres instruments ad hoc devraient être utilisés. De même, concernant le régime du contrat, il a été montré ailleurs ${ }^{44}$ comment l'ambivalence du contrat — à la fois procédure et effet conduisait la doctrine à multiplier les concepts concurrents à la nullité (inopposabilité, réputé non écrit, inefficacité, ininvocabilité, etc.) lorsque la nullité n'affectait qu'une partie des effets de l'acte. D'où une complexification inutile du droit.

Ces faiblesses aboutissent même parfois à des solutions inintelligibles tant d'ailleurs pour le justiciable que pour la doctrine. Un bon exemple de solution inintelligible pour le justiciable peut être tiré de l'arrêt Hôpital de Prades rendu en 2016 par le Conseil d'État ${ }^{45}$. Dans cet arrêt il était question d'une demande de paiement de frais d'hébergement, laquelle était formulée en référé devant le juge administratif par un établissement médico-social à l'encontre d'une usagère. Le Conseil d'État va ici juger que l'usagère «n'était pas dans une situation contractuelle vis-à-vis du service public administratif, même si elle avait conclu un contrat d'hébergement ». L'on imagine aisément la surprise des parties à cette procédure lorsqu'elles ont pris connaissance de cette décision. D'un côté le Conseil d'État leur dit qu'un contrat existe bien - puisque ce contrat est imposé par le code de l'action sociale et des familles (art. L. 311-4) — mais de l'autre il leur dit que la relation en cause n'est pas contractuelle. Comprenne qui pourra! Si nous prenons ici cet exemple c'est parce que cette situation est la conséquence directe des faiblesses du contrat dénoncées plus haut. En effet, elle résulte en partie des différences de définition retenues par le législateur et le Conseil d'État. Alors que le législateur recourt à la définition traditionnelle du contrat, en qualifiant ici cette relation de contractuelle puisque l'accord des volontés entre l'établissement et l'usager est à ses yeux créateur de normes, le Conseil d'État s'appuie quant à lui sur la représentation sociale du contrat. Si le Conseil refuse ici la qualification contractuelle c'est en effet parce qu'il reprend ici sa position de principe suivant laquelle l'usager d'un service public administratif n'est pas un contractant, cela parce que les idées d'échange économique, de

\footnotetext{
${ }^{42}$ P. Voirin obs. sous Paris, 18 déc. 1942, DC 1943, p. 36, cité par N. Balat, « Forclusion et prescription », RTD civ. 2016, p. 751-772, $\mathrm{n}^{\mathrm{o}} 7$.

${ }^{43}$ En droit de la concurrence : B. Goldman, droit commercial européen, Précis, Dalloz, $2^{\mathrm{e}}$ éd., 1971, $\mathrm{n}^{\circ} 257$; en droit des marchés financiers : Olympe Dexant - de Bailliencourt, op. cit., $\mathrm{n}^{\circ} 311$; en droit médical : L. Bloch, « Objectivisme et subjectivisme dans l'acte médical » in Mélanges en l'honneur du Professeur Jean Hauser, Lexisnexis et Dalloz, 2012, p. 752.

${ }^{44}$ Contrat ou acte juridique?, op. cit., Partie 1, Titre 1, Chapitre 1, Section 2, §2.

${ }^{45}$ CE, 3 févr. 2016, no 388643, Rec. Lebon (Tables).
} 
liberté, de formalisme et d'irrévocabilité sont trop peu présentes dans cette situation ${ }^{46}$. Les services publics administratifs sont en effet souvent gratuits, fortement encadrés par la loi, le formalisme y est généralement réduit en comparaison des services publics industriels et commerciaux et les «lois du service» permettent à la personne en charge du service de modifier la situation de l'usager sans son consentement. Il est frappant au demeurant de constater que les rares exemples - trop souvent occultés - dans lesquels la qualification contractuelle de l'usager est finalement admise sont ceux où ces idées associées à la représentation sociale du contrat sont plus nettement présentes. Tel est le cas pour le contrat d'occupation d'un $\mathrm{HLM}^{47}$, les contrats de crédits municipaux ${ }^{48}$ ou encore les contrats d'aide aux activités agricoles ou industrielles ${ }^{49}$. Dans ces situations, difficile en effet de nier la place de l'échange économique, de la liberté contractuelle (choix du logement, du montant du prêt ou de la subvention, etc.) ou encore du formalisme.

Autre exemple d'inintelligibilité, mais pour la doctrine cette fois, la jurisprudence relative à la formation du contrat. En effet, nombre d'auteurs ont exprimé leur difficulté à comprendre la logique de la Cour de cassation qui — du moins avant la réforme du 10 février 2016 - ne sanctionnait pas à leurs yeux de manière cohérente les différents actes susceptibles de former un contrat ${ }^{50}$. D'une part, ces auteurs reprochaient à la Cour de sanctionner la promesse unilatérale de la même manière que l'offre, c'est-à-dire par la simple attribution de dommages-intérêts, alors qu'à leurs yeux la promesse étant un contrat elle aurait dû être sanctionnée par une exécution forcée. D'autre part, il était reproché à la jurisprudence de la Cour de sanctionner le pacte de préférence plus durement que la promesse unilatérale, c'est-àdire par une exécution forcée, alors que le pacte devrait être à leurs yeux moins efficace que la promesse, compte tenu du caractère éventuel de la promesse qu'il renferme ${ }^{51}$. De semblables difficultés existent également en droit du travail, à propos de la jurisprudence relative à la promesse d'embauche que les auteurs peinent à comprendre et qualifient d'incohérente ${ }^{52}$. En effet, alors que certains arrêts semblent suggérer que la promesse d'embauche vaudrait contrat de travail, d'autres au contraire maintiennent une claire séparation entre ces concepts et les auteurs de conclure à l'incohérence de ces solutions. Or, il est permis de penser que le peu d'intelligibilité de ce droit positif résulte en grande partie des faiblesses théoriques dénoncées plus haut quant à la nature des actes concourant à la formation du contrat. Les actes concourant à la formation du contrat ne se voyant pas reconnus en tant que tels la qualité

\footnotetext{
${ }^{46}$ Pour qui douterait que le Conseil d'État recourt ici moins à la définition traditionnelle du contrat qu'à sa représentation sociale, l'on renverra notamment aux écrits des auteurs à l'origine de ce rejet du contrat, notamment L. Duguit, op. cit. — dont le premier argument est la trop faible liberté de l'usager —, ainsi qu'aux conclusion des rapporteurs publics rendues sous les arrêts fondateurs de ce rejet et notamment, pour la relation médicale, à celles d'A. Bacquet (AJDA, déc. 1979, p. 31 s. sous CE, Section, Tolub, 23 fév. 1979, Rec. Lebon, p. 80) qui donne un grand rôle à l'absence de liberté et à l'absence de formalisme, ainsi qu'un certain rôle au caractère économiquement peu marqué de ces relations.

${ }^{47}$ CE, OPHLM de la communauté urbaine de Strasbourg, 30 oct. 1995, no 105251, Rec. Lebon (Tables).

${ }^{48}$ CE, Rieux, 13 juin 1979, n ${ }^{\circ}$ 6861, Rec. Lebon.

${ }^{49}$ TC, Société d'approvisionnements alimentaires, 24 juin 1968, n 1917, Rec. Lebon.

${ }^{50}$ Cf. not. N. Molfessis, « De la prétendue rétractation du promettant dans la promesse unilatérale de vente ou pourquoi le mauvais usage d'un concept inadapté doit être banni », D. 2012, p. 231-237; D. Mazeaud, « Promesse unilatérale de vente : la Cour de cassation a ses raisons... », D. 2011, p. 1457-1460; L. Aynès et Ph. Stoffel-Munck, «Mars - septembre 2008 : facteurs de fragilité dans la formation des contrats », Droit \& Patrimoine, $\mathrm{n}^{\circ} 178$, p. 120-132, spé. p. 123.

${ }^{51}$ Rappr. Cass., $3^{\mathrm{e}}$ civ., 16 mars 1994, $\mathrm{n}^{\mathrm{O}}$ 91-19.797, qualifiant le pacte de préférence de «promesse unilatérale conditionnelle $»$.

${ }^{52} \mathrm{~J}$. Mouly, « Le formalisme du CDD ne s'applique pas à la promesse d'embauche : encore la distinction de la promesse et du contrat de travail », Droit social, 2016, p. 687 s. ; S. Tournaux, « La promesse d'embauche vaut contrat de travail... si le salarié l'accepte ! ", Rev. trav., 2016, p. 700 s. et, du même auteur, "Les exigences légales relatives à la forme et au contenu du CDD ne s'appliquent pas à une promesse d'embauche ", Rev. trav., 2016, p. 616 s.
} 
d'acte juridique, la doctrine peine à comprendre la nature des normes qu'ils créent et dès lors à rendre compte de la jurisprudence les concernant. Elle n'est par exemple pas en mesure de voir que la promesse unilatérale de vente ne repose que sur un seul acte juridique - la prétendue acceptation du bénéficiaire n'ajoutant aucune norme et ne pouvant donc pas être un acte juridique - de sorte qu'il n'est pas anormal théoriquement que cette promesse trouve une sanction similaire à l'offre rétractée, laquelle est aussi, avons-nous dit, un acte juridique ${ }^{53}$. Il en va de même en cas de violation du pacte de préférence où, en l'absence de rétractation par le promettant de son engagement de vendre, certes adressé à un tiers, il est normal que la jurisprudence opte pour son exécution forcée au moment où le bénéficiaire lève l'option. Quoi qu'il en soit, toutes ces jurisprudences mal comprises en doctrine ne sont pas sans conséquences pour le justiciable car cette difficulté doctrinale à rendre compte du droit positif se traduit par une insécurité juridique : la jurisprudence de la Cour de cassation n'étant pas comprise par la doctrine, les justiciables, assistés par leurs conseils, sont bien en peine d'anticiper sur les règles qui leur seront appliquées.

Deuxièmement, ces difficultés théoriques peuvent être sources de solutions mal acceptées par les justiciables. Ceci peut survenir tant dans les cas où les juges retiennent l'existence d'un contrat alors que, à suivre la définition traditionnelle du contrat, cela ne serait pas possible, que dans les cas où les juges rejettent une telle qualification alors que, au regard de cette définition, cette qualification s'imposerait.

Pour illustrer la première hypothèse l'on peut donner l'exemple de l'obligation de sécurité qui, à partir de $1911^{54}$, a été adjointe aux contrats de transport par la Cour de cassation. Lorsque les transporteurs et les membres de la doctrine sensibles à leurs arguments ont pris connaissance de ces arrêts, ils n'ont pas manqué de les critiquer en affirmant que les transporteurs n'avaient eu aucune volonté de s'engager à indemniser les personnes transportées en cas d'accident et que de telles décisions méconnaissaient la définition traditionnelle du contrat ${ }^{55}$. Compte tenu de l'autorité des décisions attachées à la Cour de cassation, ces critiques n'ont guère eu d'effets quant à l'efficacité de ces décisions. En revanche, en présence de juridiction dont le pouvoir est moins bien acceptée, en particulier certaines juridictions internationales, ceci peut avoir des conséquences sur l'acceptation de la décision. L'on peut en donner une illustration - certes à propos d'un engagement unilatéral — avec l'affaire des essais nucléaires français dans le Pacifique ${ }^{56}$, où la décision rendue par la Cour internationale de justice en 1974 a été mal reçue par le Gouvernement et la doctrine française $^{57}$, notamment parce que la Cour y prêtait au Gouvernement français l'intention de s'engager à ne plus faire d'essai nucléaire alors que le Gouvernement français, le mieux à même pour dire ce qu'il avait en tête, soutenait quant à lui ne pas avoir eu une telle intention.

Les difficultés pratiques existent également lorsque le juge écarte la qualification contractuelle, notamment en s'appuyant sur la représentation sociale du contrat plutôt que sur

\footnotetext{
${ }^{53}$ Certes, il est libre au législateur de prévoir l'irrévocabilité de la promesse et non de l'offre, comme cela est le cas dans les nouveaux textes (art. 1116 al. 3 et art. 1124 al. 2). Cependant, la difficulté à distinguer l'offre de la promesse - faudra-t-il s'en tenir à un critère formel tenant à l'apposition d'une signature sur la promesse donne à penser que la jurisprudence Consorts Cruz — relativement au refus d'exécution forcée de la promesse de vente - n'a pas dit son dernier mot. L'on ne saurait guère surpris que demain la Cour de cassation, soucieuse de protéger la liberté contractuelle, use du pouvoir qu'elle tient de l'article $12 \mathrm{du}$ CPC pour requalifier en offre des promesses et ainsi sanctionner leur violation par de simples dommages-intérêts.

${ }^{54}$ Cass., civ., 21 nov. 1911 ; sur cet arrêt cf. not. Les grands arrêts de la jurisprudence civile, tome 2, $\mathrm{n}^{\mathrm{O}} 277$.

${ }^{55}$ Cf. not. L. Josserand, «L'essor moderne du concept contractuel», Recueil sur l'étude des sources du droit en l'honneur de François Gény, Tome 2, Sirey, Paris, 1934, p. 345.

${ }^{56}$ CIJ, Affaire des essais nucléaires, Nouvelle-Zélande c/ France et Australie c/ France (2 arrêts), 20 déc. 1974, CIJ Rec., p. 253 s. et 457 s.

${ }^{57}$ J. Salmon, «Les accords non formalisés ou "solo consensu" », Annuaire français de droit international, 1999, vol. 45, p. 14 .
} 
sa définition rigoureuse. Pour revenir au droit de la santé, la solution traditionnelle du Conseil d'État de refuser à l'usager la qualification de contractant a par exemple un temps conduit le juge administratif à refuser aux établissements publics de santé le droit de recouvrer leurs créances en se fondant sur les engagements de payer signés par l'entourage du patient, privant ainsi parfois ces établissements de toute chance d'être payé ${ }^{58}$. Ce faisant, cette jurisprudence a mis mal les efforts déployés par les établissements de santé pour sécuriser le recouvrement de leurs créances. Si cette solution a pu être encore réaffirmée récemment dans l'arrêt Hôpital de Prades précité, il semble néanmoins que le Conseil d'État ait amorcé en 2014 un discret virage afin d'éviter cet inconvénient ${ }^{59}$. Pour l'instant, cependant, le revirement n'est pas total et ne couvre qu'une partie des cas dans lesquels les établissements de santé estimeraient être en droit, au regard de la définition traditionnelle du contrat, de recouvrer leur créance. D'où la persistance pour eux d'une réelle difficulté pratique trouvant son origine dans les faiblesses du contrat.

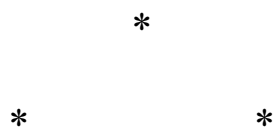

$\mathrm{Au}$ regard de l'ensemble de ces difficultés théoriques et pratiques, auxquelles de nombreuses autres pourraient être ajoutées ${ }^{60}$, il apparaît opportun d'adopter une autre définition du contrat qui aurait à cœur notamment de ne pas trop embrasser et, laissant à l'acte juridique l'aspect procédural aujourd'hui pris en charge par la conception traditionnelle du contrat, se centrerait uniquement sur un type d'effets, distinct notamment des effets unilatéraux (ou réglementaires). Dans une telle définition, le contrat serait positivement défini comme un ensemble de normes dont chacune, d'une part, serait conditionnée pour sa vigueur à l'existence d'une ou plusieurs autres normes de cet ensemble et, d'autre part, aurait pour assujetti son auteur (ou éventuellement la personne le représentant). Négativement, le contrat ne serait pas un acte juridique, qualification réservée aux actes le formant — actes n'étant pas forcément une offre et une acceptation. Les normes contractuelles pourraient ainsi cohabiter avec des normes unilatérales au sein d'un même acte juridique les ayant créées.

\footnotetext{
${ }^{58}$ CE, Section, avis, Kilou, 28 juil. 1995, no 168438, Rec. Lebon, p. 315. Sur cette question, cf. « L'usager du service public administratif hospitalier, un contractant ! ", RDSS, 2017, $\mathrm{n}^{\circ}$ 2, à paraître.

${ }^{59} \mathrm{CE}$, Centre de gestion pour expatriés et entreprises, 12 nov. 2014, $\mathrm{n}^{\mathrm{o}}$ 368876, Rec. Lebon (Tables), où l'établissement de santé est autorisé à se retourner contre un signataire d'un engagement de payer qui était en l'espèce mandaté par des patients étrangers pour s'acquitter de leurs frais. Au regard des enjeux financiers, le Conseil d'État a accepté de revenir sur sa position traditionnelle.

${ }^{60} \mathrm{Cf}$. les nombreux autres exemples de difficultés pratiques mentionnés dans Contrat ou acte juridique ?, op. cit.
} 